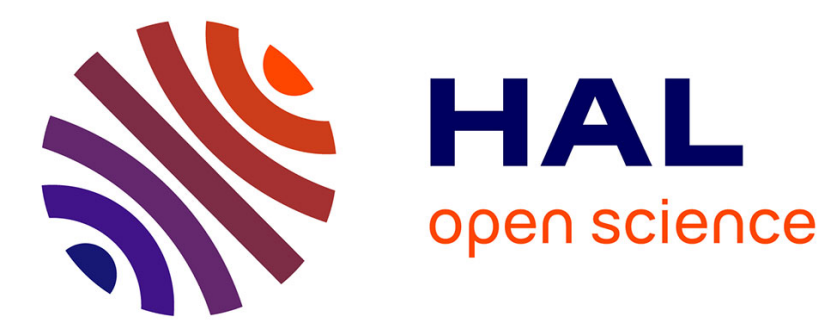

\title{
A new analytical torque formula for axial field permanent magnets coupling
}

Bastien Dolisy, Smail Mezani, Thierry Lubin, Jean Lévêque

\section{To cite this version:}

Bastien Dolisy, Smail Mezani, Thierry Lubin, Jean Lévêque. A new analytical torque formula for axial field permanent magnets coupling. IEEE Transactions on Energy Conversion, 2015, 8 p. 10.1109/TEC.2015.2424159 . hal-01164388

\section{HAL Id: hal-01164388 \\ https://hal.science/hal-01164388}

Submitted on 16 Jun 2015

HAL is a multi-disciplinary open access archive for the deposit and dissemination of scientific research documents, whether they are published or not. The documents may come from teaching and research institutions in France or abroad, or from public or private research centers.
L'archive ouverte pluridisciplinaire HAL, est destinée au dépôt et à la diffusion de documents scientifiques de niveau recherche, publiés ou non, émanant des établissements d'enseignement et de recherche français ou étrangers, des laboratoires publics ou privés. 


\title{
A new analytical torque formula for axial field permanent magnets coupling
}

\author{
B. Dolisy, S. Mezani, T. Lubin, J. Lévêque
}

\begin{abstract}
In this paper, we present a simple and accurate analytical expression to compute the torque of axial-field magnetic couplings. The torque expression is obtained by solving the three-dimensional (3D) Maxwell equations by the method of separation of variables. Here we adopt the assumption of linearization at the mean radius, the problem is then solved in $3 \mathrm{D}$ Cartesian coordinate (we neglect the curvature effects). To show the accuracy of the torque formula, the results are compared with those obtained with 3D finite-element simulations, and experimental tests. As the proposed formula needs very low computational time and depends directly on the geometrical parameters, it is used for a design optimization using multiobjective genetic algorithms.
\end{abstract}

Index Terms - Genetic algorithms, magnetic coupling, permanent magnets, torque transmission, 3D analytical model.

\section{INTRODUCTION}

$\mathrm{M}$ agnetic couplings (or couplers) can transmit a torque without mechanical contact. This is very interesting for applications requiring isolation between two different atmospheres. They can be used in the naval propulsion [1] for torque transmission between motors and propellers, or in chemical industry for health constraints. In addition, the absence of mechanical contact increases the lifetime of the system and reduces noise, vibrations and mechanical friction losses. Moreover, it provides natural protection against overloads.

Magnetic couplings can have axial or radial flux topologies (Fig. 1). They both consist of two rotors, each of which is composed of an array of permanent magnets alternately magnetized along the $\theta$-direction. The two rotors present the same number of pole pairs ( $p=6$ in Fig. 1 ).

The axial-flux topology is studied in this paper. It consists of two similar rotors facing each other. As shown in Fig. 2, the air-gap between the two rotors is noted $e$.

The magnets are sector shaped with a thickness (along $z$ direction) noted $h$ for both rotors. The inner and outer radii are respectively noted $R_{\text {in }}$ and $R_{\text {out }}$.

The authors are with the Groupe de Recherche en Electrotechnique et Electronique de Nancy, Université de Lorraine, 54506 Nancy, France (e-mail: ’b-dolisy@hotmail.fr).

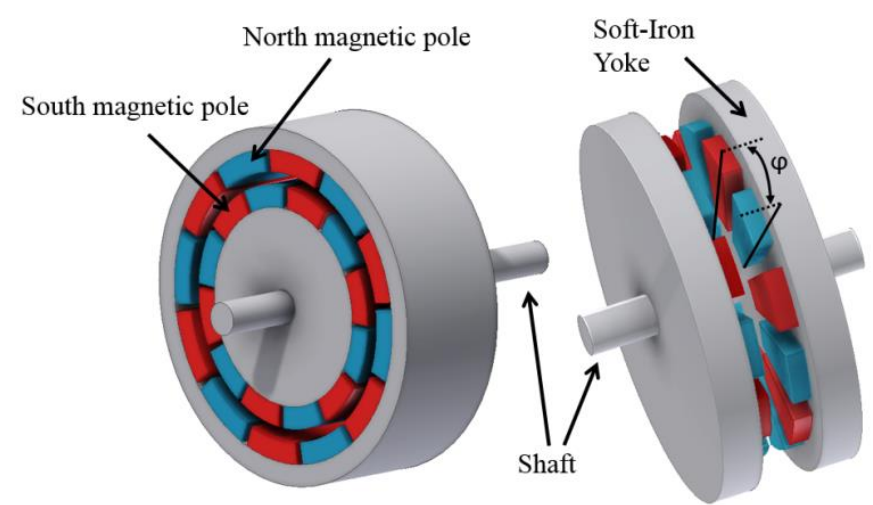

(a) Radial flux

Fig. 1 Topologies of rotating magnetic couplings $(p=6)$.

(b) Axial flux

The magnet angular opening to pole opening ratio is noted $\alpha$ and varies between 0 and 1 . The angular lag (load angle) between the two rotors is noted $\varphi$.

The electromagnetic computation of magnetic couplings is carried out using several methods. The Finite-element (FE) method is so far the preferred method of analysis. Indeed, it leads to accurate results taking into account the non-linearity of magnetic materials and the actual coupler geometry [2-4]. The main drawback of FE methods is the long computation time and the lack of flexibility. It is therefore unsuitable for optimization purposes which require many repetitive computations. In order to reduce the computation time, analytical models can be developed by solving the partial differential equations (PDE) arising from Maxwell's equations. It is necessary to make some assumptions regarding the linearity of magnetic materials and geometry simplification [5-9]. Usually, the problem is solved under a two-dimensional (2D) approximation which, in some situation like in axial field couplers, results in a $30 \%$ overestimation of the torque compared to 3D FE prediction [10-12]. Threedimensional analytical models for magnetic couplings have been proposed in the literature [6],[13] and [14]. Biot-Savart like formulas are used to determine the magnetic field distribution in ironless structures (magnets in free space).

The method of images could be used to consider infinitely permeable iron walls but the computation time increases. Recently, it has been shown that Fourier analysis can be used to solve 3D problems with ferromagnetic parts [7]. 


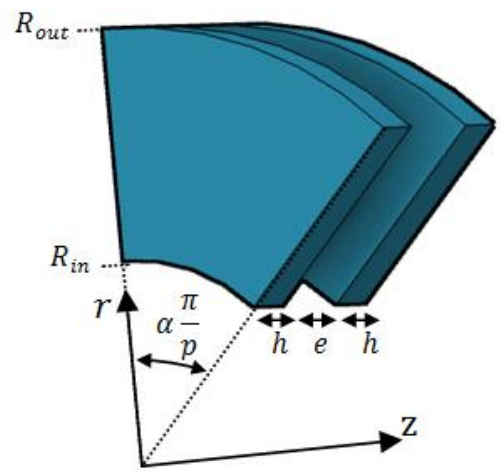

Fig. 2 Dimension of the permanent magnets of an axial flux coupler topology $\varphi=0$ (iron yokes not shown).

In [5], the authors developed a 3D analytical model to compute the no load flux in axial-field permanent magnet synchronous machine. In this method (also called sub-domain method), it is necessary to numerically solve an algebraic system of linear equations to calculate the Fourier coefficients.

Hence, even keeping its analytical formalism, the "fully analytical" meaning of the sub-domain method is somewhat lost. Nevertheless, in terms of computation time, such a method remains more efficient than a FE analysis.

In this paper, the sub-domain method is used to analytically determine the magnetic field distribution in the axial magnetic coupling shown in Fig. 1b. A new and purely analytical expression for the torque evaluation is then derived from the 3D solution. The proposed torque formula, which depends directly on the physical and geometrical parameters, is obtained by solving the PDEs in 3D Cartesian coordinates by assuming a linearized geometry at the mean radius (we neglect the curvature effects). We also consider an infinite permeability of the iron yokes.

The torque expression is obtained in two steps:

- Firstly, we only consider the magnets on one side of the coupling (the magnets on the other rotor are turned off). Then, we compute the magnetic field by using a magnetic scalar potential formulation.

- Secondly, using the analogy between the electrostatic and the magnetostatic fields, the magnetic force acting on the magnets placed on the opposite side is obtained by using the equivalent electrostatic Lorentz force.

To analyze the accuracy of the proposed torque formula, the results are compared to those obtained from 3D finite-element simulations and from experimental investigations. Finally, the analytical formula is used for a genetic algorithm multiobjective optimization of the coupler.

\section{ANALYTICAL MODEL}

As stated above, the torque expression will be derived using the analogy that exists between the electrostatic and the magnetostatic fields.
A. Magnetic force from the electrostatic-magnetostatic analogy

For simplicity, let us consider, in free space, an electrostatic uniform surface charge density $\sigma_{\mathrm{s}}\left(\mathrm{C} / \mathrm{m}^{2}\right)$, subjected to an electric field $\vec{E}$ [16]. The Lorentz force (N) exerted on $\sigma_{\mathrm{s}}$ is

$$
\overrightarrow{F_{s}}=\sigma_{s} \iint_{S} \vec{E} d S
$$

where $\mathrm{S}$ is the surface which carries $\sigma_{\mathrm{s}}$.

From the magnetostatic point of view, it is usual to use an equivalent magnetic surface charge $\sigma_{\mathrm{m}}$ in $\mathrm{A} / \mathrm{m}$ [7],[11],[14],[15],[16]. Unlike $\sigma_{\mathrm{s}}$, the magnetic charge $\sigma_{\mathrm{m}}$ doesn't have any physical meaning. However, it is introduced for modelling purposes in which it usefully replaces some magnetic field sources (magnets, current carrying solenoids,...).

The magnetic force $(\mathrm{N})$ which is analogous to the electrosatic one, given by (1), is then obtained by

$$
\overrightarrow{F_{m}}=\sigma_{m} \iint_{S} \vec{B} d S
$$

Here, $\mathrm{S}$ is the surface which carries $\sigma_{\mathrm{m}}$.

The force expressions (1) and (2) show that the electrostaticmagnetostatic analogy links the electric field $\vec{E}$ to the $\vec{B}$ field (called flux density).

Concerning the studied magnetic coupler, all what we need to compute the force is the magnet's magnetic surface charge on one rotor and the magnetic field created by the magnets of the second rotor (the magnets on the first rotor are turned off).

Furthermore, expression (2) which uses Lorentz force in free space gives, for our coupler, the right values of the force along the $\mathrm{x}$ and $\mathrm{y}$ directions only (no other material than air in these two direction). However, since ferromagnetic materials are present in the z-direction, (2) will not give the right value of the force and we have to use Maxwell stress tensor or virtual work methods.

To deal with the presence of iron media an equivalent surface charge of the ferromagnetic material could be introduced [15].

\section{B. Magnetic field due the magnets of one rotor}

The iron-yokes have an infinite permability. Hence, the magnetic field is null in the iron parts.

The boundary condition on the iron interface is then

$$
\vec{n} \times \vec{H}=0
$$

where $\overrightarrow{\mathbf{n}}$ is the outward normal to the considered surface and $\overrightarrow{\boldsymbol{H}}$ the magnetic field strength.

Rare-earth permanent magnets have a relative permeability close to that of air $\left(\mu_{r}=1\right)$. 
The studied coupler doesn't contain any current source. To solve the magnetostatic problem, it is then more convenient to use a magnetic scalar potential (noted $\Phi$ ) formulation (such as $\vec{H}=-\nabla \Phi$ ). In the different media, the flux density $\vec{B}$ is given by

Air region: $\quad \vec{B}=-\mu_{0} \nabla \Phi$

Magnets region: $\quad \vec{B}=\mu_{0}(-\nabla \Phi+\vec{M})$

where $\vec{M}$ is the magnetization of the magnet.

To simplify the analysis, we make the assumption of linearization at the mean radius, so the curvature effect is not considered. However, this allows to solve the problem in a Cartesian coordinates system, which is simpler than to solve the problem in cylindrical coordinates where special functions appear [5]. The validity of the linearized model is discussed later in the paper.

Fig. 3 shows the problem to solve after linearization. The main dimensions of the linearized coupler are

$$
\begin{gathered}
l_{m y}=\frac{R_{\text {out }}-R_{\text {in }}}{2} ; l_{m x}=\alpha D_{x} \\
D_{x}=\frac{\pi R_{\text {mean }}}{2 p} ; R_{\text {mean }}=\frac{R_{\text {out }}+R_{\text {in }}}{2}
\end{gathered}
$$

Due to the alternate polarity along the $x$-direction, only one pole is considered with anti-periodic boundary conditions along $\mathrm{x}$.

A second anti-periodic condition is applied at the external boundaries on the y-coordinate. This condition is a fictitious but a necessary one in order to get a solution. Nevertheless, by setting $D_{y}>>l_{m y}$, this anti-periodic condition leads to $\vec{B}=$ 0 at $y= \pm D_{y}$, which is of course a more realistic physical condition (Usually, $D_{y}=2 l_{m y}$ allows to obtain accurate results).

As stated above, the whole resolution domain contains magnet and air regions, Fig.4.

Domain I $(0 \leq z \leq h)$ corresponds to the magnet region of height $h$. The magnetization vector is noted $\vec{M}=M_{z}(x, y) \overrightarrow{e_{z}}$ and it is obtained by expanding the magnetization into a double Fourier series along $\mathrm{x}$ and $\mathrm{y}$-directions (Fig. 5).

$$
M_{z}(x, y)=\sum_{n=1}^{\infty} \sum_{m=1}^{\infty} M_{n, m} \cos \left(w_{n} x\right) \cos \left(w_{m} y\right)
$$

with $\quad M_{n, m}=\frac{16 B_{r}}{\mu_{0} n m \pi^{2}} \sin \left(w_{n} l_{m x}\right) \sin \left(w_{m} l_{m y}\right)$

$$
w_{n}=\frac{n \pi}{2 D_{x}} ; w_{m}=\frac{m \pi}{2 D_{y}}
$$

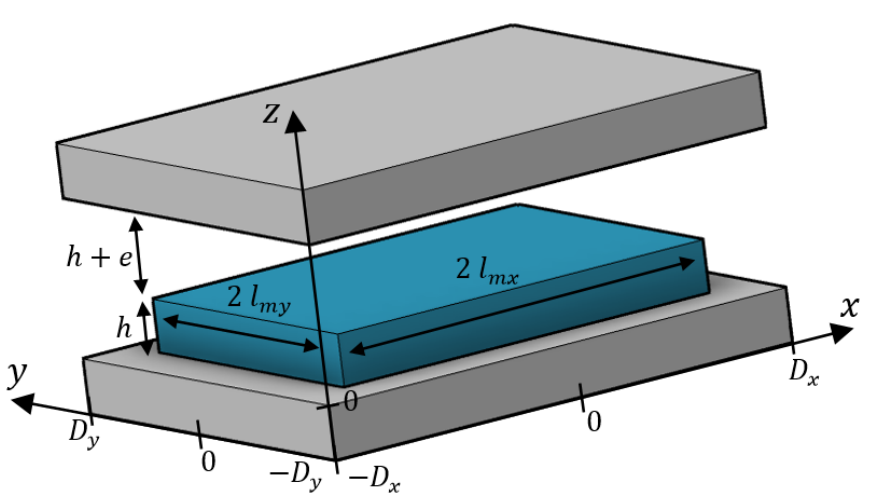

Fig. 3 Dimensions of one magnet pole after linearization (axis of rotation, located at $y=-R_{\text {mean }}$ not shown).

where $n, m$ are odd integers and $B_{r}$ is the residual flux density of the permanent magnets. Notice that the magnetization given by (7) is divergence free $\nabla \cdot \vec{M}=0$.

Domain II $\left(h \leq z \leq h_{t}\right)$ is composed of the actual air-gap and the second magnet (whose magnetization is turned off).

The magnetic scalar potential is noted $\Phi_{I}$ in domain I and $\Phi_{I I}$ in domain II. $\Phi_{I}$ and $\Phi_{I}$ are the solution of Laplace equation

$$
\begin{gathered}
\nabla^{2} \Phi_{I}=\nabla \cdot \vec{M}=0 \\
\nabla^{2} \Phi_{I I}=0
\end{gathered}
$$

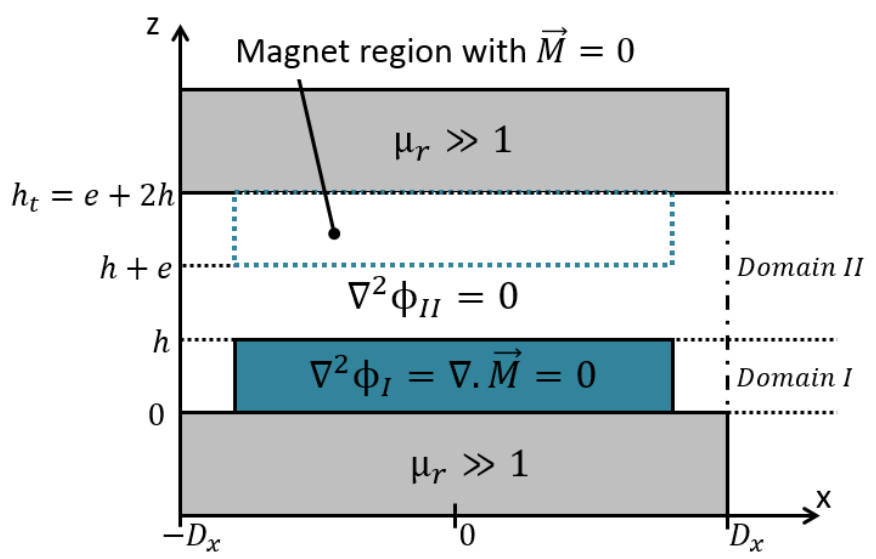

Fig. 4 Domains and equations in the plan (x,z).

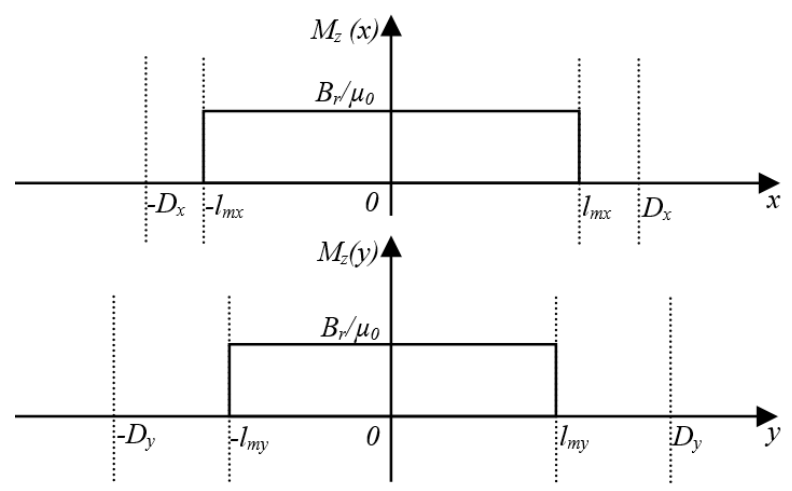

Fig. 5 Magnetization $\mathrm{Mz}$ as a function of $\mathrm{x}$ and $\mathrm{y}$ (domain $\mathrm{I}$ ). 
By considering the anti-periodic boundary conditions along the $\mathrm{x}$ and $\mathrm{y}$ coordinates, the use of the method of separation of variables leads to the following form solutions for $\Phi_{I}$ and $\Phi_{I I}$

$$
\begin{aligned}
\Phi_{I}(x, y, z)=\sum_{\mathrm{n}=1}^{\infty} \sum_{\mathrm{m}=1}^{\infty}\left[A_{I} e^{k z}+B_{I} e^{-k z}\right] \\
\times \cos \left(\mathrm{w}_{\mathrm{n}} x\right) \cos \left(\mathrm{w}_{\mathrm{m}} y\right) \\
\Phi_{I I}(x, y, z)=\sum_{\mathrm{n}=1}^{\infty} \sum_{\mathrm{m}=1}^{\infty}\left[A_{I I} e^{k z}+B_{I I} e^{-k z}\right] \\
\times \cos \left(\mathrm{w}_{\mathrm{n}} x\right) \cos \left(\mathrm{w}_{\mathrm{m}} y\right)
\end{aligned}
$$

with $k=\sqrt{w_{n}^{2}+w_{m}^{2}}$

The coefficients $A_{I}, B_{I}, A_{I I}$ and $B_{I I}$ are obtained using the interface and boundary conditions.

The Boundary condition in domains I and II are set at $\mathrm{z}=0$ and $\mathrm{z}=\mathrm{h}_{\mathrm{t}}$, respectively. These conditions state that the tangential magnetic field components $\mathrm{H}_{\mathrm{x}}$ and $\mathrm{H}_{\mathrm{y}}$ are zero (iron boundaries). This leads to

$$
\begin{gathered}
A_{I}+B_{I}=0 \\
\mathrm{~A}_{\mathrm{II}} \mathrm{e}^{\mathrm{k} \mathrm{h} t}+\mathrm{B}_{\mathrm{II}} \mathrm{e}^{-\mathrm{kh}_{t}}=0
\end{gathered}
$$

Interface conditions between domains I and II are set at $\mathrm{z}=\mathrm{h}$. Domain I and II have the same magnetic permeability $\left(\mu_{\mathrm{r}}=1\right)$, so the normal flux density $\left(\mathrm{B}_{\mathrm{z}}\right)$ and the tangential magnetic fields $\left(\mathrm{H}_{\mathrm{x}}\right.$ and $\left.\mathrm{H}_{\mathrm{y}}\right)$ of the domain I and II will be equal at $\mathrm{z}=\mathrm{h}$. The two following expressions arise

$$
\begin{gathered}
A_{I} e^{k h}+B_{I} e^{-k h}-A_{I I} e^{k h}-B_{I I} e^{-k h}=0 \\
A_{I} e^{k h}-B_{I} e^{-k h}-A_{I I} e^{k h}+B_{I I} e^{-k h}=\frac{M_{n, m}}{k}
\end{gathered}
$$

Finally, the coefficients $\mathrm{A}_{\mathrm{I}}, \mathrm{B}_{\mathrm{I}}, \mathrm{A}_{\mathrm{II}}$ and $\mathrm{B}_{\mathrm{II}}$ are calculated by solving an algebraic system of linear equations arising from (10) and (11). They are given by

$$
\begin{gathered}
A_{I}=-B_{I}=\frac{M_{n, m}\left(e^{2 h k}-e^{2 h_{t} k}\right)}{2 k e^{h k}\left(e^{2 h_{t} k}-1\right)} \\
A_{I I}=-\frac{M_{n, m} \sinh (h k) e^{-h_{t} k}}{2 k \sinh \left(h_{t} k\right)} \\
B_{I I}=\frac{M_{n, m} \sinh (h k) e^{h_{t} k}}{2 k \sinh \left(h_{t} k\right)}
\end{gathered}
$$

\section{Equivalent surface charge density of the second PM rotor}

The equivalent surface charge density of a magnet (Coulombian model) with uniform magnetization is given by

$$
\sigma_{m}=\vec{M} \cdot \vec{n}
$$

Where $\vec{M}=M \overrightarrow{e_{z}}=B_{r} / \mu_{0} \overrightarrow{e_{z}}$ is the magnetization vector and $\vec{n}$ represents the outward normal to the considered surface. This dot product has to be performed on all the external surfaces of the magnet volume.

Fig.6 shows a rectangular permanent magnet with a uniform magnetization in the $z$-direction. From (13), the magnet is then represented by two surface charge densities $\sigma_{+}$and $\sigma_{-}$.

In our problem, the surface charge density $\sigma_{+}=M$ is located at $z=h_{t}$ and the surface charge density $\sigma_{-}=-M$ at $z=h+$ e.

\section{Torque expression}

The force is computed using (2) where the integration is performed on the surfaces carrying $\sigma_{+}$and $\sigma_{-}$. However, according to the boundary condition (3), the tangential components $B_{x}$ and $B_{y}$ of the flux density are null on the charged surface $\sigma_{+}$(at $z=h_{t}$ ), so the forces that contribute to torque $\left(\mathrm{F}_{\mathrm{x}}\right.$ and $\left.\mathrm{F}_{\mathrm{y}}\right)$ also vanishes. Hence, the integation is only performed on the charged surface $\sigma_{-}($at $z=h+e)$.

The axis of rotation (the shaft axis) is parallel to the $\mathrm{Oz}$ axis. This axis has constant coordinates noted $\left(\mathrm{x}_{0}, \mathrm{y}_{0}\right)$ in the (Oxyz) reference frame. The $\mathrm{z}$-component of the torque is then obtained by

$$
T=\int_{-l_{m x}+X_{i}}^{l_{m x}+X_{i}} \int_{-l_{m y}}^{l_{m y}}\left[\left(x-x_{0}\right) f_{y}-\left(y-y_{0}\right) f_{x}\right] d x d y
$$

The variable $X_{i}$ in (14) corresponds, in cartesian coordinates, to the angular lag (load angle) $\varphi$ between the two rotors of the coupling. $X_{i}$ and $\varphi$ are related by $\varphi=X_{i} / R_{\text {mean }}$.

Notice that the maximum (pull-out) torque is obtained for a position $X_{i}=D_{x}$.

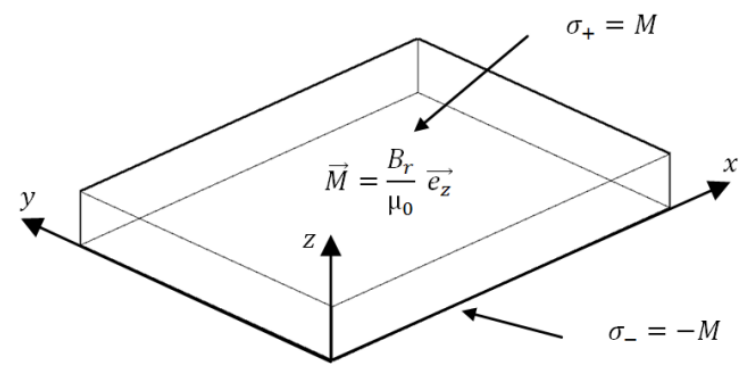

Fig. 6 Equivalent surface charge density for a rectangular permanent magnet. 
In (14), $f_{x}$ and $f_{y}$ represent the force densities in $\left(N / \mathrm{m}^{2}\right)$ obtained by replacing the flux density expression (4) in the force expression (2).

$$
\begin{aligned}
& f_{x}=-\left.\mu_{0} \sigma_{-} \frac{\partial \Phi_{I I}(x, y, z)}{\partial x}\right|_{z=h+e} \\
& f_{y}=-\left.\mu_{0} \sigma_{-} \frac{\partial \Phi_{I I}(x, y, z)}{\partial y}\right|_{z=h+e}
\end{aligned}
$$

The force density $f_{y}$ being symetrical along the y-direction, the corresponding torque obtained by integration between $-l_{m y}$ and $l_{m y}$ vanishes. Hence, for $2 \mathrm{p}$ poles, and $\mathrm{y}_{0}=-\mathrm{R}_{\text {mean }}$ the torque expression (14) becomes

$$
\begin{array}{r}
T=2 p \mu_{0} \sigma_{-} \int_{-l_{m x}+X_{i}}^{l_{m x}+X_{i}} \int_{-l_{m y}}^{l_{m y}}\left(y+R_{\text {mean }}\right) \\
\times\left.\frac{\partial \Phi_{I I}}{\partial x}\right|_{z=h+e} d x d y
\end{array}
$$

Finally, from (9) and (12) an analytical closed-form expression for the torque is obtained after integration of (17)

$$
\begin{gathered}
T=\sum_{n=1}^{N} \sum_{m=1}^{V} \frac{128 p B_{r}^{2} R_{\text {mean }}}{\pi^{2} \mu_{0} n m k w_{m}} \sin ^{2}\left(\alpha n \frac{\pi}{2}\right) \sin ^{2}\left(w_{m} l_{m y}\right) \\
\times \frac{\sinh ^{2}(k h)}{\sinh \left(k h_{t}\right)} \sin (n p \varphi)
\end{gathered}
$$

where $N$ and $V$ are the number of harmonic terms used for the torque calculation.

Another useful quantity to compute is the flux over a pole surface. Indeed, this allows sizing the yoke thickness through the flux conservation law. This flux is higher on the iron surface ( $\mathrm{z}=0$ for instance) at no-load $(\varphi=0)$. The integration of $\mathrm{B}_{\mathrm{z}}(\mathrm{x}, \mathrm{y}, \mathrm{z}=0)$, due to the two PMs rotors, over the pole surface gives then

$$
\begin{aligned}
& \psi_{m}{ }^{N} \sum_{n=1} \sum_{m=1}^{V} \frac{128 B_{r} R_{\text {mean }} D_{y}}{n^{2} m^{2} \pi^{3} p} \\
& \times\left(\frac{\sinh \left(k\left(h-h_{t}\right)+\sinh (k h)\right.}{\sinh \left(k h_{t}\right)}+1\right) \\
& \times \sin \left(w_{n} D_{x}\right) \sin \left(w_{n} l_{m x}\right) \sin ^{2}\left(w_{m} l_{m y}\right)
\end{aligned}
$$

The thickness of iron yokes $\left(\mathrm{h}_{\text {iron }}\right)$ can be determined using flux conservation law. If $\mathrm{B}_{\mathrm{ymax}}$ is the maximal wished value of the flux density in the yoke cross section area, then

$$
h_{\text {iron }}>\frac{\psi_{m}}{2 B_{\text {ymax }}\left(R_{\text {out }}-R_{\text {in }}\right)}
$$

$\mathrm{B}_{\mathrm{ymax}}=1.2-1.4 \mathrm{~T}$ is a reasonable value to avoid saturation of mild steel.

\section{EVALUATION OF THE TORQUE FORMULA}

In this section, we analyze the accuracy of the developed torque formula whose results are compared to those issued from 3D FE computations (Comsol multiphysics ${ }^{\circledR}$ ) carried out on the actual cylindrical coupling.

The FE model is implemented under Comsol Multiphysics software. The "no current" module which uses a magnetic scalar potential $(\phi)$ formulation has been used. Mild steel $\mathrm{B}(\mathrm{H})$ curve is used for the yokes. However, the yoke thickness is choosen to avoid magnetic saturation. Hence, a linear model with a relative permeability value equals to 1000 is used for the ferromagnetic material so the computation time is reduced without any loose of accuracy.

The 3D mesh consists of 300808 1st order tetrahedral elements leading to solve a global algebraic system having 416231 degrees of freedom. An infinite box surrounds the studied system in order to set Dirichlet boundary conditions $(\phi=0)$. Only 1 pole of the coupler is considered with antiperiodic boundary conditions in the azimuthal direction.

In [11] and [12], the authors have constructed a prototype axial field coupling and developed a 2D formula to evaluate the torque. The main parameters of this prototype are given in Table I and a photograph is shown Fig. 7.

The corresponding experimental and analytical results also serve to evaluate the torque formula (17).

The measurements are carried out as follows, Fig.7 (more details can be found in [11] and [12]).

The relative angular position $\delta$ was measured using an encoder with a resolution of 4096 steps per revolution (precision of 0.088 degrees). The relative error on the full measured angle range of $15^{\circ}$ does not exceed $0.6 \%$.

The static torque was measured thanks to precise weights (the weight scale is within $10 \mathrm{~g}$ precision) suspended to a rod (1 meter length) locked to one rotor, the other being fixed. The torque is obtained by multiplying the weight by the rod length (lever arm).

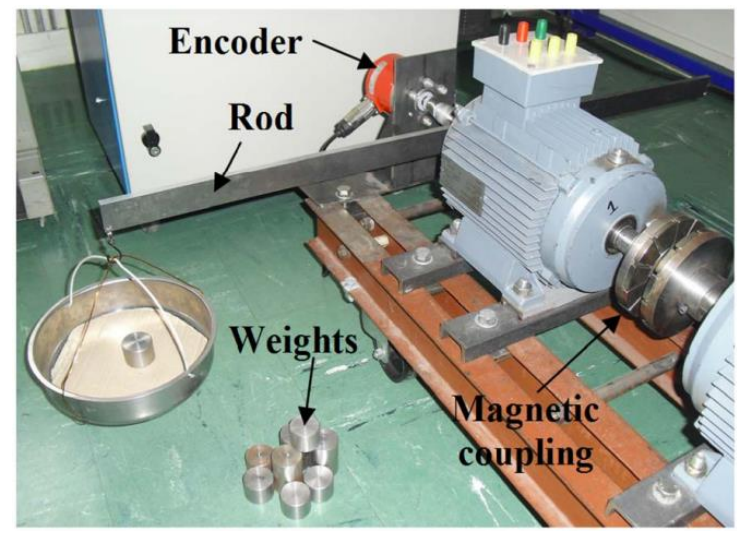

Fig. 7 Axial flux magnetic coupling prototype [11],[12]. 
TABLE I

DIMENSIONS OF THE CONSTRUCTED MAGNETIC COUPLING [11],[12]

\begin{tabular}{|l|l|l|l|}
\hline \multicolumn{1}{|c|}{ Parameters } & \multicolumn{1}{|c|}{ Description } & Unit & \multicolumn{1}{c|}{ Value } \\
\hline $\mathbf{R}_{\text {out }}$ & Outer radius of magnets & $\mathrm{mm}$ & 60 \\
\hline $\mathbf{R}_{\text {in }}$ & Inner radius of magnets & $\mathrm{mm}$ & 30 \\
\hline $\mathbf{h}$ & Magnet thickness & $\mathrm{mm}$ & 7 \\
\hline $\mathbf{h}_{\text {iron }}$ & Iron yoke thickness & $\mathrm{mm}$ & 10 \\
\hline $\mathbf{e}$ & Thickness of the air gap & $\mathrm{mm}$ & variable \\
\hline $\boldsymbol{\alpha}$ & Magnet to pole opening ratio & - & 0.9 \\
\hline $\mathbf{p}$ & Number of pole pairs & - & 6 \\
\hline $\mathbf{B}_{\mathbf{r}}$ & Residual induction of permanent magnets & $\mathrm{T}$ & 1.25 \\
\hline
\end{tabular}

Hence, the absolute error on the torque measure is about $0.1 \mathrm{Nm}(0.01 \times 9.81 \times 1)$ which is very low in regard to the torque values in presence here.

Hence, all the measured torque values are rounded to the closest first digit (ie. $60.58 \mathrm{Nm}$ becomes $60.6 \mathrm{Nm}$ ).

The thickness of the iron yoke have been chosen to avoid saturation. The flux computed analytically using (18) is about $0.67 \mathrm{mWb}$ whereas the $3 \mathrm{D}$ FE computation gives $0.68 \mathrm{mWb}$. This shows the accuracy of the analytical expression (18). The mean flux density on the iron yoke surface is $0.95 \mathrm{~T}$.

\section{A. Influence of the number of harmonic terms $N$ and $V$ in evaluating the torque}

The torque expression (17) contains a double sum which depends on the number of harmonic terms $N$ and $V(N$ and $V$ correspond respectively to the number of harmonic terms in the $x$-direction and in the $y$-direction. A certain number of harmonic terms has to be used to get a stable solution. Of course, this number has to be as low as possible to ensure a good accuracy and the lowest possible computation time.

We compare here the results obtained using (18) and the experimental ones which correspond to the axial coupling having the parameters of Table I.

Fig. 8 shows the error on the pull-out torque evaluation vs. the number of harmonics $N$ and $V$. The error is defined as

$$
\varepsilon_{\%}=100 \frac{T_{\text {experimental }}-T}{T_{\text {experimental }}}
$$

where $T_{\text {experimental }}=60.6 \mathrm{Nm}$ is the measured pull-out torque for an air gap e $=4 \mathrm{~mm}$.

If we only consider the fundamental term for the $\mathrm{x}$-direction $(N=1)$, we can observe in Fig. 8 that the calculation slightly overestimates the torque $\left(\varepsilon_{\%}=-7 \%\right)$. If we add a supplementary harmonic $(N=3)$, the computation provides very accurate result with an error estimate lower than $2 \%$.

This good accuracy requires to consider only two harmonic terms along the y-direction $(V=3)$ to take into account the edge effects (3D effects).

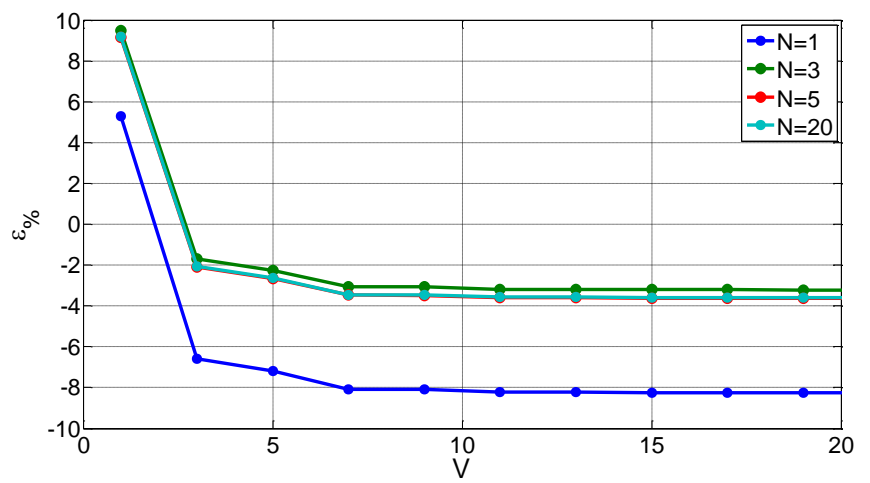

Fig. 8 Error on the torque prediction vs. the numbers of harmonics $\mathrm{N}$ and V.

Anyhow, even when taking $\mathrm{N}=20$ and $\mathrm{V}=20$, the computation time remains very low (less than $12 \mathrm{~ms}$ ). So we can conclude that we need very few harmonic terms in order to have a good precision.

\section{B. Comparison to experimental and $3 D F E$ results}

In this section, we compare experimental measurements for the static torque with numerical and analytical computations. An analytical formula which is derived in [11] using a 2D analytical model (mean radius model and first harmonic approximation) is given by (21). This formula doesn't take into account the edge effects.

$$
\begin{aligned}
T=\frac{16}{3 \pi} \frac{B_{r}{ }^{2}}{\mu_{0}} R_{\text {out }} & \left(1-\left(\frac{R_{\text {in }}}{R_{\text {out }}}\right)^{3}\right) \\
& \times \sin ^{2}\left(\alpha \frac{\pi}{2}\right) \frac{\sinh ^{2}(a)}{\sinh (2(1+v) a)} \\
& \times \sin (p \varphi) \\
\text { with } \quad a= & p \frac{h}{R_{\text {mean }}} \quad \text { and } \quad v=\frac{e}{2 h}
\end{aligned}
$$

Figs. 9 show the results of the torque calculation for two airgap values $(e=4 \mathrm{~mm}$ and $e=9.5 \mathrm{~mm}$ ) obtained by all the methods in use. Whetever the air-gap, we note that the analytical expression (17) used with $(\mathrm{N}=20, \mathrm{~V}=20)$, the 3D FE model as well as the measurements give very close torque values.

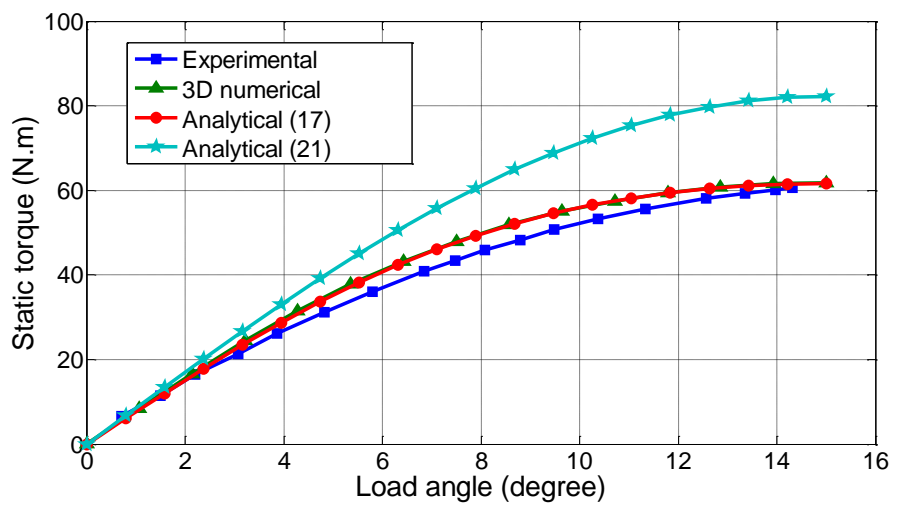

(a) $e=4 \mathrm{~mm}$ 


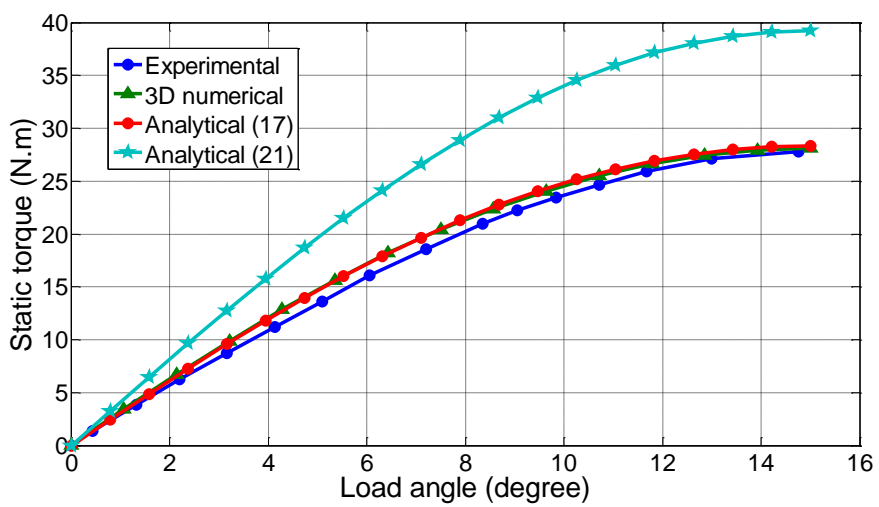

(b) $e=9.5 \mathrm{~mm}$

Fig. 9 Static torque of the magnetic coupling obtained with several methods in use - (18) used with $\mathrm{N}=\mathrm{V}=20$.

However, the analytical expression (21) overestimates the maximum torque by approximately $30 \%$. This clearly shows the necessity to consider the radial fringing effects in axial field couplings.

\section{Curvature effects}

In order to address the limits of the analytical formula regarding the curvature effects, the analytical computation (linearized coupling) are compared to 3D finite element simulations (actual cylindrical topology) for several dimensions of the magnet. For a given air gap, the error introduced by the linearization assumption depends on the radial excursion $R_{\text {out }}-R_{\text {in }}$ and on the mean pole pitch which is equal to $($ Rout + Rin $) \pi / 2 p$. (see Fig.2). To analyze the influence of these parameters, we introduce a dimensionless number $\lambda$ which allows comparing the pole pitch and the radial excursion.

$$
\lambda=p \frac{1-\beta}{1+\beta} \text { with } \beta=\frac{R_{\text {in }}}{R_{\text {out }}}
$$

It was possible to analyze the problem using each parameter as a variable while keeping the others constant. But finally, we found that this single parameter $\lambda$ led to the same conclusions.

Two computations are performed: the first one uses 3D FEM to obtain the torque of the cylindrical coupling.

The second one uses the analytical expression (17) to calculate the torque of the linearized cylindrical coupling (we have considered $\mathrm{N}=20$ and $\mathrm{V}=20$ ).

For a magnet height $h=10 \mathrm{~mm}$ and for two values of the air gap ( $e=5 \mathrm{~mm}$ and $e=10 \mathrm{~mm}$ ), we vary $R_{\text {out }}, R_{\text {in }}$ and $p$ in the following intervals:

- $\quad R_{\text {out }}=[0.1 \mathrm{~m}$ to $0.3 \mathrm{~m}]$ with a step of $0.1 \mathrm{~m}$ (three values);

- $\quad R_{\text {in }}=\left[0.3 \times R_{\text {out }}\right.$ to $\left.0.8 \times R_{\text {out }}\right]$ with a step of $0.1 \times$ $R_{\text {out }}$ (six values);

- $\quad p=[2$ to 8] with a step of 1 (seven values);
This corresponds to 504 combinations (252 for each topology).

Fig. 10 presents the error on the torque calculation between the analytical expression (17) and the 3D FEM. It can be seen that the error doesn't exceed $3 \%$ for an airgap of $5 \mathrm{~mm}$. This error rises to $6 \%$ when the airgap value is $10 \mathrm{~mm}$ but this concerns very few points.

These results clearly validate the linearization hypothesis. Regarding the computation time for the 504 combinations, the 3D finite-element simulations have taken several hours while the analytical computations needed less than one second.

\section{Optimization of the coupler using genetic algorithms}

Genetic Algorithms (GA) are widely used as a robust and effective tool in optimization problems. We use here the NSGA2 implementation of GA under Matlab [17].

The airgap in axial coupling could be easily varied to deal with several operating situations (i.e. hermetic isolation of 2 media with variable axial lengths). Hence, we propose to deal with the multiobjective optimization of the following problem:

- The design variables vector is: $X=\left(p ; R_{\text {out }} ; R_{i n} ; h ; \alpha\right)$,

- The objective function are: maximize $\left(\mathrm{T}_{2}\right)$, minimize $\left(\mathrm{M}_{\mathrm{PM}}\right)$,

- The constraints are: $\mathrm{T}_{1}=100 \mathrm{Nm}, \mathrm{B}_{\text {mean }}<0.9 \mathrm{~T}$.

The objective $T_{2}(\mathrm{Nm})$ is the torque for an air gap $\mathrm{e}=10 \mathrm{~mm}$ and the objective $\mathrm{M}_{\mathrm{PM}}$ corresponds to the total mass of the PMs.

The remanence of the PMs is $\mathrm{B}_{\mathrm{r}}=1.25 \mathrm{~T}$ and their mass density is equal to $7600 \mathrm{~kg} / \mathrm{m}^{3}$.

The constraints $T_{1}$ corresponds to the desired torque for $\mathrm{e}=5 \mathrm{~mm}$ and the constraint $\mathrm{B}_{\text {mean }}$ is set to limit the iron saturation. $\mathrm{B}_{\text {mean }}=\psi_{\mathrm{m}} / \mathrm{S}_{\mathrm{p}}$ is the average flux density over the iron pole surface $S_{p}=\pi\left(R_{\text {ext }}^{2}-R_{\text {in }}^{2}\right) / 2 p$, and $\psi_{\mathrm{m}}$ is given by (18).

The bounds of the variables in the vector $X$ are: $p=2-20$; $\mathrm{R}_{\text {out }}=20-100 \mathrm{~mm} ; \mathrm{R}_{\text {in }}=10-90 \mathrm{~mm} ; \mathrm{h}=2-10 \mathrm{~mm}, \alpha=0.2-0.9$.

The optimization procedure uses 100 individuals evolving during 100 generations, but 50 generations are enough to reach a stable solution. The computation time for 100 generations is about 1 minute.

Topological groups having different pole-pair values appear in the obtained Pareto front of Fig.11.

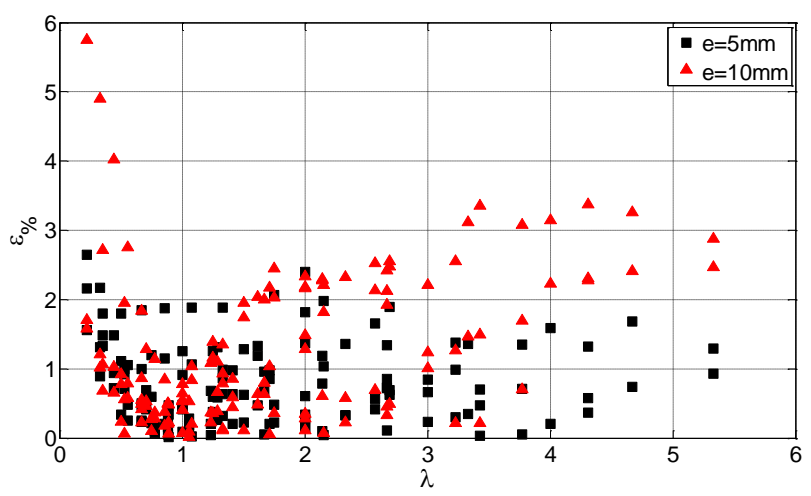

Fig. 10 Error on the torque calculation between the analytical expression (linearized geometry) and the 3D FE model (cylindrical topology). 


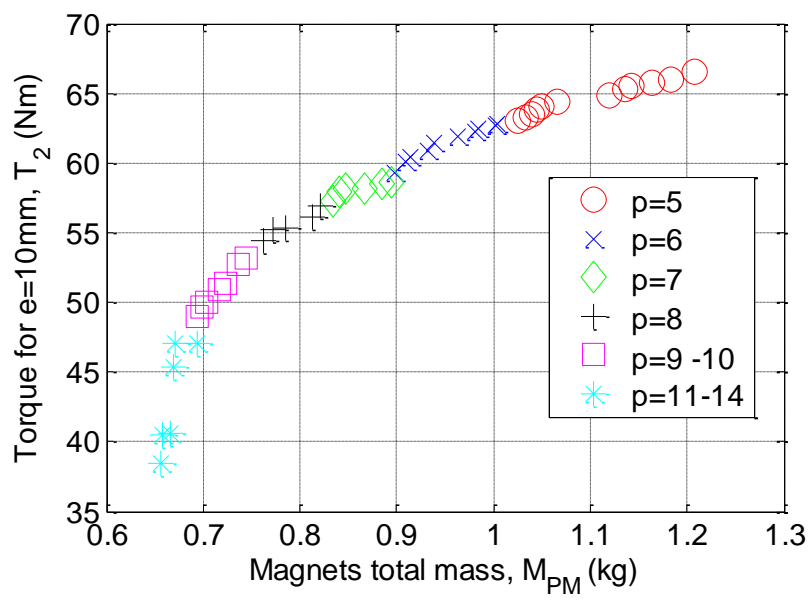

Fig. 11 Pareto front: Torque $T_{2}$ vs. PMs mass $\mathrm{M}_{\mathrm{PM}}$.

As expected, the highest numbers of $\mathrm{p}$ lead to the lowest PM mass but also to lower torque $\mathrm{T}_{2}$. It can be observed that to increase $\mathrm{T}_{2}$ from 50 to $65 \mathrm{Nm}$ (30\% increase) we need a $70 \%$ increase of the PM mass (from 0.7 to $1.2 \mathrm{~kg}$ ).

Notice that many of the solutions of Fig.11 have checked by the 3D FE model and the error in the worst case is less than $3 \%$. This, again, demonstrates the effectiveness and accuracy of the analytical formula.

\section{CONCLUSION}

A new analytical expression to compute the torque of a PM axial field magnetic coupling has been derived. This expression has been obtained thanks to 3D magnetostatic analytical. By introducing a surface charge density, the torque computation used the electrostatic-magnetostatic analogy to evaluate the Lorentz force. We have shown that the proposed torque formula is very accurate and computationally very efficient. Thus, it has been used to optimize the studied coupler by a multiobjective genetic algorithm.

\section{ACKNOWLEDGMENT}

The authors would like thank the DGA (Direction Générale de l'Armement) for funding this work.

\section{REFERENCES}

[1] J. F. Charpentier, N. Fadli, and J. Jennane, "Study of Ironless Permanent Magnet Devices Being Both a Coupling and an Axial Bearing for Naval Propulsion,” IEEE Trans. Magn., vol. 39, no. 5, pp. 3235-3237, May 2003.

[2] T. F. Chan, W. Wang, and L.L. Lai, "Performance of an axial-flux permanent magnet synchronous generator from 3-D finite-element analysis," IEEE Trans. Energy Convers., vol. 25, no. 3, pp. 669-676, Sept 2010.Letter Symbols for Quantities, ANSI Standard Y10.5-1968.

[3] W. Yao, J. Jin, P. T. Krein, M. P. Magill, "A Finite-Element-Based Domain Decomposition Method for Efficient Simulation of Nonlinear Electromechanical Problems," IEEE Trans. Energy Convers., vol. 29, no. 2, pp. 309-319, Jun. 2014.

[4] Wang Yu Lin, Liu Pin Kuan, Wu Jun, and Ding Han, "Near-Optimal Design and 3-D Finite Element Analysis of Multiple Sets of Radial Magnetic Couplings," IEEE Trans. Magn., vol. 44, no. 12, pp. $4747-$ 4753, Dec. 2013.
[5] O. de la Barrière, S. Hlioui, H. Ben Ahmed, M. Gabsi, and M. LoBue "3-D Formal Resolution of Maxwell Equations for the Computation of the No-Load Flux in an Axial Flux Permanent-Magnet Synchronous Machine,” IEEE Trans Magn, vol. 48, no. 1, pp. 128-136, Jan. 2012.

[6] J. P. Yonnet, S. Hemmerlin, E. Rulliere and G. Lemarquand, "Analytical calculation of permanent magnet couplings," IEEE Trans. Magn., vol. 29, no. 6, pp. 2932-2934,Jun. 1993.

[7] J. P. C. Smeets, T. T. Overboom, J. W. Jansen, and E. A. Lomonova, "Mode-Matching Technique Applied to Three- Dimensional Magnetic Field Modeling,” IEEE Trans. Magn, vol. 48, no. 11, pp. 3383-3386, Mar. 2012.

[8] J. F. Charpentier and G. Lemarquand, "Study of Permanent-Magnet Couplings with Progressive Magnetization Using an Analytical Formulation," IEEE Trans. Magn., vol. 35, no. 5, pp. 4206-4217,1999.

[9] Jang-Young Choi, Hyeon-Jae Shin, Seok-Myeong Jang, and Sung-Ho Lee, "Torque Analysis and Measurements of Cylindrical Air-Gap Synchronous Permanent Magnet Couplings Based on Analytical Magnetic Field Calculations," IEEE Trans. Magn., vol. 49, no. 7, pp. 3921-3924, Jul. 2013

[10] J. Azzouzi, G. Barakat, and B. Dakyo, "Quasi-3-D analytical modeling of the magnetic field of an axial flux permanent-magnet synchronous machine," IEEE Trans. Energy. Convers, Vol 20, no. 4, pp.746-752, Dec. 2005.

[11] T. Lubin, S. Mezani and A. Rezzoug, "Simple Analytical Expressions for the Force and Torque of Axial Magnetic Couplings," IEEE Trans. Energy. Convers, Vol 27, no. 2, pp.536-546, Jun. 2012.

[12] T. Lubin, S. Mezani and A. Rezzoug, "Experimental and Theoretical Analyses of Axial Magnetic Coupling under Steady-State and Transient Operations," IEEE Trans. Ind. Electronics, Vol 27, no. 2, pp.4356-4365, Aug. 2014.

[13] E. P. Furlani and M. A. Knewtson, "A three-dimensional field solution for permanent-magnet axial-field motors," IEEE Trans. Magn., vol. 33, no. 3, pp. 2322-2325, May. 1997.

[14] R. Ravaud, G. Lemarquand, V. Lemarquand, and C. Depollier, "Permanent magnet couplings: Field and torque three-dimensional expressions based on the Coulombian model," IEEE Trans. Magn., vol. 45, no. 4, pp. 1950-1958, Apr. 2009.

[15] D. T. E. H. van Casteren, J. J. H. Paulides, and E. A. Lomonova, "3-D Numerical Surface Charge Model Including Relative Permeability: The General Theory," IEEE Trans. Magn., vol. 50, no. 11, pp. 8204704, Nov. 2014.

[16] E. Durand, Electrostatique et Magnétostatique, Masson \& Cie, Paris, France, 1953, $774 \mathrm{p}$.

[17] S. Lin, NGPM - A NSGA-II Program in Matlab v1.4, Available online: http://uk.mathworks.com/matlabcentral/fileexchange/31166-ngpm-ansga-ii-program-in-matlab-v1-4

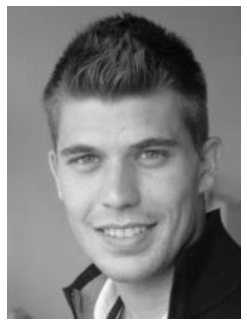

Bastien Dolisy was born in Sarreguemines, France, in 1989. He received the M. S. degree from the University of Lorraine, Nancy, France in 2012 and the $\mathrm{Ph} . \mathrm{D}$. degree from the University of Lorraine, Nancy, France, in 2015 . He is currently a lecturer at the University of Lorraine (Nancy), France, at the Groupe de Recherche en Electrotechnique et Electronique de Nancy where his research interests include the applications of superconductors in electromechanical devices.

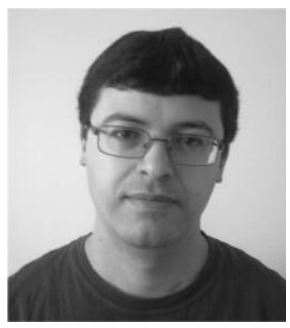

Smail Mezani was born in Algiers, Algeria, in 1974. He received the engineer diploma and the magister degree fro $m$ the University of Sciences and Technology Houari Boumediene, Algiers, Algeria in 1996 and 1999 respectively. He obtained the Ph.D. degree from the Institut National Polytechnique de Lorraine, France, in 2004. He is currently a lecturer at the University of Lorraine (Nancy), France, at the Groupe de Recherche en Electrotechnique et Electronique de Nancy where his research interests include the applications of superconductors in electromechanical devices. 


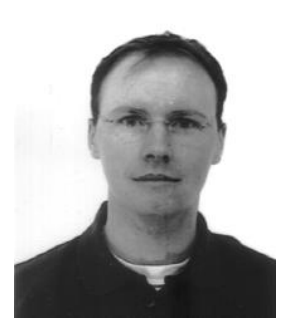

Thierry Lubin was born in Sedan, France, in 1970. He received the M.S. degree from the University Pierre et Marie Curie, Paris 6, France in 1994 and the Ph.D. degree from the University of Lorraine, Nancy, France, in 2003. He is currently a lecturer of Electrical Engineering at the University of Nancy at the Groupe de Recherche en Electrotechnique et Electronique de Nancy. His interests include modeling and control of electrical machines, and applied superconductivity in electrical devices.

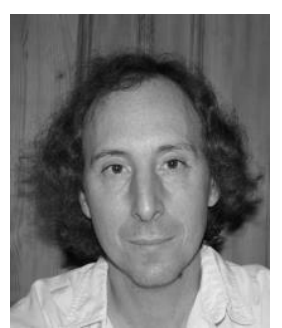

Jean Lévêque was born in Angers, France, in 1963. In 1993, he received the Ph.D. degree in electrical engineering from the University of Grenoble, Grenoble, France. He joined the laboratory GREEN in the University de Lorraine, Nancy, France, in 1993. His research interests include two main topics, the application of the superconductor's material in electrical engineering, especially superconducting motor, fault current limiter, and electrical characterization, and modelization of superconducting material. 